Para citar este artículo: Maas dos Anjos, R. \& Ribeiro Freyesleben, L.E. (2020). El cambio climático como mito: política contra la ciencia. Sostenibilidad: económica, social y ambiental, 2, 1-10. https://doi.org/10.14198/Sostenibilidad2020.2.01

\title{
El cambio climático como mito: política contra la ciencia
}

\section{Climate change as a myth: politics against science}

\author{
Rafael Maas dos Anjos \\ Universidade do Vale do Itajaí, Brasil \\ rafamaas@gmail.com \\ https://orcid.org/0000-0003-4931-7159
}

Luiz Eduardo Ribeiro Freyesleben

Universidade do Vale do Itajaí, Brasil

lef11192@tjsc.jus.br

\section{RESUMEN}

Este artículo estudia la proliferación en el entorno político del discurso que afirma que el cambio climático es un mito. Los periódicos de mayor difusión se han utilizado como fuentes de investigación. En ellos, ilustres figuras políticas de los países analizados expresan la idea, o sugieren, que la acción antrópica no es decisiva para el cambio climático en el planeta. Uno de los argumentos más frecuentes reside en que las medidas recomendadas para combatir el calentamiento global y, en consecuencia, el cambio climático, tienen objetivos indirectos relacionados con favorecer a ciertos países y crear barreras para el desarrollo de otros. El estudio tiene como objetivo confrontar estas afirmaciones de los representantes del gobierno con las compilaciones de datos científicos contenidos en los repositorios de entidades especializadas. La prevalencia de uno $\mathrm{u}$ otro entendimiento no solo es relevante a nivel académico, por el contrario, puede guiar las acciones de los gobiernos, comprometiendo las firmas de nuevos tratados ambientales o implicando la salida de otros ya firmados. Se pretende así evaluar si tiene sentido la tesis negacionista.

Palabras clave: cambio climático, calentamiento global, sostenibilidad. 


\begin{abstract}
This article studies the proliferation in the political environment of the discourse that climate change is a myth. Newspapers with wide circulation are used as sources of research. In them, prominente politicians in their countries express the idea - or suggest - that anthropic action is not decisive for the change of climate on the planet. One of the most frequent arguments is that the recommended measures to combat global warming have obscure objectives related to favoring certain countries and creating barriers to the development of others. The study aims to confront these assertions of government representatives with the compilations of scientific data contained in the repositories of specialized entities. The prevalence of one or other understanding is not only relevant at the academic level, on the contrary, it can guide the actions of governments, compromising the signatures of new environmental treaties or implying the exit of others already signed. Thus, it is intended to evaluate, if there is sense in the negationist thesis.
\end{abstract}

Keywords: climate change, global warming, sustainability.

\title{
1. Introducción
}

Los cambios en el clima de la Tierra han sido objeto de numerosos estudios en las universidades e institutos de investigación más reconocidos. El tema surgió casi como un lugar común en el mundo científico. Hasta entonces, era necesario conocer los detalles del problema para establecer acciones y estrategias transnacionales para combatirlo. Lo que parecía prescindible era profundizar el debate sobre la premisa principal: la ocurrencia real de un cambio climático antrópico capaz de provocar el colapso del mundo.

Sin embargo, en los últimos años esta certeza ha sido cuestionada. Surgieron voces contra las medidas implementadas o propuestas por la comunidad internacional. En opinión de los opositores a estas iniciativas de protección ambiental, tendrían el objetivo oculto de favorecer a algunos países y evitar el auge económico de otros. Tenemos un dilema.

El propósito de este texto es contribuir a la evolución del diálogo y el debate sobre el calentamiento global, la ciencia y la política, para alcanzar el camino intermedio, donde el equilibrio siempre es bienvenido, es sostenible. Metodológicamente, este texto utiliza el método deductivo, utilizando la técnica de investigación bibliográfica, abordando el tema de la sostenibilidad en el mundo político y científico.

\section{Análisis del estado de la cuestión}

Las declaraciones de figuras políticas que han salido a la luz recientemente sugieren la existencia de una conspiración ideológica a gran escala que amenazaría la soberanía de los estados. Investigar si la tesis negacionista tiene sentido no es una mera curiosidad académica, ya que puede influir en la sociedad y guiar acciones futuras del gobierno, como la negativa a firmar nuevos tratados ambientales o la ruptura de tratados ya firmados.

El cuestionamiento de la veracidad de los estudios sobre el cambio climático ya no se puede atribuir a los usuarios anecdóticos de las redes sociales, ya que cada vez más proviene de personalidades que ocupan funciones relevantes en la sociedad y en los gobiernos. La voz de los miembros del aparato estatal trae la semilla de la institucionalización del discurso del escepticismo sobre las amenazas ambientales, lo que lleva a frenar las medidas preventivas y 
de contención de daños. La vacilación en este asunto, peor aún, la acción opuesta, es particularmente peligrosa porque los principales estudios muestran que, en la carrera por la supervivencia, más de la humanidad que del planeta, comenzamos con un enorme retraso.

Existe una aparente tendencia mundial de convertir las certezas científicas en noticias falsas al servicio de los grupos de conspiración. Los ataques contra la ciencia han venido incluso de aquellos que teóricamente tratan con el conocimiento, con pretensiones intelectuales. La paradoja en esta postura radica en el hecho de que, al hacerlo, estimulan una aversión a la cultura, en un movimiento que tiende a ver algo parásito e inútil en el conocimiento y la reflexión.

Para lograr el propósito de este artículo, seleccionamos algunos ejemplos en los que figuras prominentes (políticos en sus mandatos o ilustres voces inspiradoras de gobiernos recientes) se opusieron a las conclusiones de investigadores de diferentes campos, como la biología, la geografía y la física.

De hecho, examinar el discurso de los negadores no es una tarea simple. Con la misma facilidad con la que usan frases atrapadas, en forma de ondas de choque, se desmienten rápidamente y atribuyen el discurso principal a la prensa u otros oponentes reales $\mathrm{o}$ imaginarios. Reescribir hechos, negarlos, reafirmarlos y negarlos, suena como un método de desinformación aplicable a todas las ciencias.

Las declaraciones de los políticos o las personas a su alrededor se distribuyeron en varios canales de comunicación, todos dirigidos a la ciencia. Aquí se hace una breve compilación, para ejemplificar el creciente choque entre la investigación científica y el discurso político.

Un conocido filósofo brasileño contemporáneo, considerado una referencia intelectual del gobierno, publicó un video en la Web en 2012, pero que en 2019 volvió a circular en las redes sociales, en el que atribuía a Isaac Newton la práctica de la magia disfrazada de ciencia, enfatizando sus prácticas masónicas. El elemento esotérico oculto también dominaría el pensamiento de Bacon y Galileo. El heliocentrismo, en su opinión, sería una mentira a la que nos acostumbramos a repetir (Carvalho, 2012). Einstein diseñó la reconstrucción de toda la física solo para evitar tener que admitir que la Tierra no se mueve. Según él, Humboldt ya había predicho que no habría pruebas de la exactitud de la teoría copernicana. La cultura científica heredada del Renacimiento, para él, entroniza un gran fraude (UOL, 2019), en gran parte debido a su proximidad al pensamiento esotérico.

El presidente de los Estados Unidos, después de afirmar que el cambio climático era una farsa, volvió a la carga, pero más cauteloso, pero no menos controvertido. The New York Times publicó la noticia de que para el mandatario "[...] algo está pasando. Algo está cambiando y volverá a cambiar", lo que denota su creencia en una reversión espontánea. Hay otro discurso intrigante que se le atribuye: "No creo que haya un engaño. Creo que probablemente haya una diferencia. Pero no sé si es hecho por el hombre". Por lo tanto, se puso en duda la acción antrópica, así como la exención de los investigadores: "Tendrías que mostrarme a los científicos, porque tienen una agenda política muy grande" (Friedman, 2015).

Según otro periódico, el británico The Independent, Trump sostuvo que no creía en el cambio climático por la acción humana, porque el agua y el aire de America "en este momento están en su mejor registro de limpieza" (Embury-Dennis, 2018). 
La agencia de noticias Bloomberg recupera una declaración del mandatario de los Estados Unidos en noviembre de 2012 que enfatiza el contenido político del calentamiento global: "el concepto de calentamiento global fue creado por y para los chinos con el fin de hacer que la fabricación estadounidense no sea competitiva" (Dlouhy, 2018).

Y recientemente, en la 50a reunión del Foro Económico Mundial, en Davos, Suiza, el líder estadounidense llamó a los activistas climáticos "profetas de destrucción" y dijo, cuando se le preguntó acerca de su posición sobre el cambio climático, que él cree mucho en el medio ambiente y que el medio ambiente "es muy importante para mí", concluyendo que "el mayor deber de una nación es con sus propios ciudadanos. Él dijo: "solo cuando los gobiernos pongan a sus ciudadanos en primer lugar, serán invertidos en sus futuros nacionales" (Deutsche Welle, 2020).

Esta opinión parece ser compartida por el Ministro de Relaciones Exteriores de Brasil, que clasificó el cambio climático como parte de una trama de "marxistas culturales", con el objetivo de "reprimir el crecimiento económico de los países capitalistas democráticos y promover el crecimiento de China" (García, 2019). El canciller describe la ciencia climática como un dogma que ignora la evidencia que desmiente el reclamo de temperaturas en aumento y concentraciones más altas de $\mathrm{CO}_{2}$ en la atmósfera. En su percepción, este dogma creado sirve para justificar el aumento del poder regulador de los Estados sobre la economía y el poder de las instituciones internacionales sobre los Estados nacionales y sus pueblos (Martínez, 2018).

Hay quienes prefieren un silencio elocuente sobre el tema. Es el caso del partido VOX, cuyo espacio en la escena política española ha sorprendido y atraído la atención de los analistas. En las cien medidas contenidas en su programa electoral, no se menciona el cambio climático, ni siquiera el medio ambiente, a diferencia de las otras asociaciones. Sin embargo, cuatro puntos del programa se refieren al desarrollo de un plan de energía para obtener la autosuficiencia energética. La única pista, bastante vaga, que puede sugerir una leve preocupación ambiental está en el punto 38, que habla de energía barata, sostenible, eficiente y limpia. El punto 54 desmiente la parte final al establecer el objetivo de reducir los impuestos directos sobre el diésel, lo que lo permite cubrir los fertilizantes, herbicidas y plásticos, a fin de reducir los costos de producción (Roca, 2019).

Ya en 2007, el Informe de Desarrollo Humano de las Naciones Unidas, titulado "La lucha contra el cambio climático: solidaridad frente a un mundo dividido", advirtió que la inacción pone a prueba un principio básico de iluminismo (o ilustración): que, gracias al progreso humano, el futuro siempre será mejor que el pasado. El cambio climático tiende a afectar más fuertemente a las partes más pobres de la población mundial (Programa de las Naciones Unidas para el Desarrollo [PNUD], 2008, p. 1). La economía, utilizada en repetidas ocasiones como justificación de la inercia política sobre el tema ambiental, parece irónica e igualmente otra víctima. La mayor frecuencia de sequías, tormentas e inundaciones sin duda significará la pérdida de cultivos, un aumento en el precio de los alimentos y mayores gastos para la recuperación de la infraestructura.

El mismo estudio acusa la aproximación del llamado "punto de inflexión", con la activación de eventos no lineales e impredecibles. Destaca, por ejemplo, que las concentraciones de dióxido de carbono exceden el nivel natural de los últimos 650.000 años y, después de esa escala, podría significar un aumento de $5{ }^{\circ} \mathrm{C}$ en la temperatura mundial, un cambio comparable al que ocurrió en la última edad de hielo, en la que una buena parte de Europa y 
América del Norte estaban bajo un kilómetro de hielo. El documento enfatiza la necesidad de un marco multilateral que implicará respuestas políticas rápidas, con la presencia clave de países que batieron récords en emisiones. Este marco podría construirse cuando el Protocolo de Kyoto agotara su misión en 2012 (PNUD, 2008, p. 3). El Informe de 2007 se coloca aquí en un plan excepcional para mostrar que los eventos no se desarrollaron como se imaginó. El esfuerzo multilateral no llegó, por el contrario, muy pronto fue boicoteado por la salida de los Estados Unidos, el mayor contaminador, del Acuerdo de París.

Continuando con el Informe de Desarrollo Humano de las Naciones Unidas, se puede ver que, si el mundo necesitara una mayor coordinación entre países y una mayor armonía entre políticos y científicos, dado el tiempo limitado para los cambios necesarios, lo que presenciamos fue un revés. Esto es fácil de entender de uno de los extractos del Informe, reproducido aquí:

"Hay razones para sentirse optimista. Hace cinco años [2002], el mundo todavía estaba estancado en la discusión sobre si hubo o no un cambio climático y si fue inducido por humanos o no. De hecho, el escepticismo reinaba sobre el fenómeno. Hoy la controversia es cosa del pasado y las posiciones escépticas están cada vez más aisladas. La cuarta evaluación realizada por el Grupo Intergubernamental de Expertos en Intercambio Climático estableció un consenso científico agonizante de que el cambio climático es real y que se origina en la actividad humana. Casi todos los gobiernos del mundo comparten este consenso. Además, tan pronto como se publicó el Informe Stern sobre La economía del cambio climático, la mayoría de los gobiernos también aceptaron que la solución al problema del cambio climático es más factible que los costos de la inacción" (PNUD, 2008, p. 5).

Yendo a una fecha más reciente, el Intergovernmental Panel on Climate Change (IPCC), también vinculado a la ONU, con 195 países miembros y que reúne a unos 2.000 investigadores, proporciona importantes subsidios para una evaluación seria (The Intergovernmental Panel on Climate Change, IPCC, 2018). En un estudio de 2018, el IPCC anticipa los impactos que un aumento de más de $1,5^{\circ} \mathrm{C}$ en la temperatura puede tener en el planeta, en las esferas más diversas, cubriendo la intensidad y frecuencia de eventos extremos, daños al ecosistema y la biodiversidad, reducción de recursos naturales, riesgos para la seguridad alimentaria y la industria del turismo. La investigación dirigida por académicos de las regiones más diversas del mundo sugiere una revisión del límite de contención del calentamiento, previamente indicado como $2{ }^{\circ} \mathrm{C}$. En cuanto a la extinción de especies, prevé lo siguiente:

"Se proyecta que el número de especies que se prevé pierdan más de la mitad de su rango geográfico determinado climáticamente a $2{ }^{\circ} \mathrm{C}$ de calentamiento global $(18 \%$ de los insectos, $16 \%$ de las plantas, $8 \%$ de los vertebrados) se reducirá al $6 \%$ de los insectos, $8 \%$ de plantas y $4 \%$ de vertebrados a $1,5^{\circ} \mathrm{C}$ de calentamiento (confianza media). Los riesgos asociados con otros factores relacionados con la biodiversidad, como incendios forestales, fenómenos meteorológicos extremos y la propagación de especies invasoras, plagas y enfermedades, también serían menores a $1,5{ }^{\circ} \mathrm{C}$ que a $2{ }^{\circ} \mathrm{C}$ de calentamiento (alta confianza), apoyando una mayor persistencia de los servicios del ecosistema" (HoeghGuldberg, Jacob y Taylor, 2018, p. 179).

Después de detallar la severa interferencia en el régimen de lluvias, el estudio aborda la influencia en el nivel de los océanos: 
"El GMSLR [Global mean sea level rise - aumento medio global del nivel del mar] proyectado para $1,5^{\circ} \mathrm{C}$ de calentamiento global tiene un rango indicativo de $0,26-0,77 \mathrm{~m}$, en relación con 1986-2005, (confianza media). Un aumento menor del nivel del mar podría significar que hasta 10,4 millones menos de personas (con base en la población mundial de 2010 y suponiendo que no haya adaptación) estarían expuestas a los impactos del aumento del nivel del mar a nivel mundial en 2100 a $1,5^{\circ} \mathrm{C}$ en comparación con a $2{ }^{\circ} \mathrm{C}$. Una tasa más lenta de aumento del nivel del mar permite mayores oportunidades de adaptación (confianza media). Existe una gran confianza en que el aumento del nivel del mar continuará más allá de 2100. Existen inestabilidades tanto en las capas de hielo de Groenlandia como en la Antártida, lo que podría generar aumentos de varios metros en el nivel del mar en escalas temporales de siglo a milenio. Existe una confianza media en que estas inestabilidades podrían desencadenarse en torno a $1,5{ }^{\circ} \mathrm{C}$ a $2{ }^{\circ} \mathrm{C}$ del calentamiento global" (HoeghGuldberg et al., 2018, p. 178).

A medida que el negacionismo se vuelve más difícil de sostener, negado por los hechos y la sucesión de catástrofes, la tendencia es la metamorfosis. Su segunda fase es la aceptación parcial de la realidad y la exaltación del argumento económico: las medidas de contención aniquilarían los empleos. Paul Krugman escribe con dureza:

Las afirmaciones apocalípticas sobre el costo de reducir las emisiones son especialmente extrañas dado el tremendo progreso tecnológico en energía renovable: los costos de la energía eólica y solar se han desplomado. Mientras tanto, las centrales eléctricas de carbón se han vuelto tan poco competitivas que la administración Trump quiere subsidiarlas a expensas de una energía más limpia (Krugman, 2018).

Y completa: "En realidad, por supuesto, la negación climática nunca ha tenido mucho que ver ni con la lógica ni con la evidencia; como dije, los negadores están discutiendo claramente de mala fe. Realmente no creen lo que dicen” (Krugman, 2018).

La relevancia del conocimiento científico es bastante obvia en este tema. La belleza de la ciencia radica en su compleja objetividad. Aunque se trata de fenómenos cuya explicación depende de una investigación profunda, no admite divergencias. No utiliza la maleabilidad de las palabras; no se basa en juegos semánticos para justificar conclusiones previamente establecidas para satisfacer sus propios intereses. La teoría científica puede fallar, pero al menos se basa en los rigores del laboratorio. No teme la observación exhaustiva y la repetición para, si es necesario, reconocer sus propios errores y descartar las líneas de investigación equivocadas. El malabarismo engañoso de la lengua vernácula no le sienta bien, un escenario en el que la política se deleita.

La ciencia es un intento de entender el mundo que rechaza la celebración de la ignorancia observada en nuestro tiempo. Sus valores son similares a los de la democracia. Presupone el libre intercambio de ideas, el constante cuestionamiento de sus variables, la negación del secreto $y$, aunque sea falible, es una buena herramienta para evitar que seamos engañados (Sagan y Druyan, 2006, pp. 43-44 y 59). A menudo, es cierto, sus conclusiones no son muy agradables para el mundo de la política. Como escribió Sagan, "dado que la ciencia nos lleva a comprender cómo es realmente el mundo, en lugar de como nos gustaría que fuera, sus hallazgos pueden, en todos los casos, no ser inmediatamente comprensibles o satisfactorios" (Sagan y Druyan, 2006, p. 47). En cualquier caso, "la ciencia nos advierte contra los 
peligros introducidos por las tecnologías que cambian el mundo, especialmente el medio ambiente de que dependen nuestras vidas. La ciencia proporciona un sistema esencial de alerta temprana" (Sagan y Druyan, 2006, p. 58).

El astrónomo estadounidense antes mencionado sostiene que el estudio científico está equipado con una serie de elementos capaces de identificar falacias nacidas de la necesidad de justificar argumentos contradictorios y/o superficiales. Basado en Sagan, podemos resumir las adicciones más frecuentes de la siguiente manera:

a) Ataque contra el argumentador: la descalificación del interlocutor conduce al rechazo puro y simple de los argumentos, ya que ni siquiera son dignos de reflexión, debido a su origen.

b) Argumento de autoridad: otro obstáculo para la reflexión sobre lo que se dice, como si las supuestas cualidades extraordinarias del hablante fueran suficientes para la validez del argumento.

c) Apelar a la ignorancia: proclamar la profundización innecesaria de hipótesis y la investigación de resultados.

d) Petición de principio: presupone las respuestas deseadas, basadas en premisas igualmente deseadas, elegidas para justificarlas.

e) Selección parcial de observaciones: restringirse a enunciar uno o unos pocos hechos sobre un fenómeno favorable a la conclusión a la que se espera llegar.

f) Invocación de excepciones como regla general: la exaltación de fenómenos aislados como representativos del conjunto, por ejemplo: el frío intenso en una región determinada, en un período específico, contradiría el calentamiento global.

g) Tergiversación de las estadísticas: cerca del elemento anterior, consiste en utilizar datos cuantitativos aislados, no representativos del conjunto, para refutar una hipótesis.

h) Análisis a corto plazo: considerar el retraso de tiempo más pequeño puede distorsionar los resultados; la no variación de la temperatura promedio en una fracción insignificante de tiempo, por ejemplo, puede llevar a la conclusión de que no hay peligro de calentamiento anormal del planeta.

i) Palabras equívocas: use la polisemia de las palabras para exagerar algunos aspectos y enmascarar otros, como el uso del término "producto fitosanitario" en lugar de "pesticida" (Sagan y Druyan, 2006, pp. 244-249).

Valorar a quienes dedican la mayor parte de su tiempo a determinar y cuantificar los resultados y sus causas es un requisito previo para cualquier debate público con un mínimo de seriedad.

Los ejemplos mundiales son preocupantes. Tome el ejemplo de Australia. En 2019, el país más grande de Oceanía registró $49,5^{\circ} \mathrm{C}$, con registros de sequía y calor (Euronews, 2019). Recientemente en 2020, en los primeros días, Australia fue consumida por incendios graves, 
posiblemente agravados por el intenso calor y la sequía récord de 2019, ingredientes que facilitan la propagación de las llamas (Eler, 2020).

Ahora, parece que incluso la naturaleza ha perdido la paciencia con la política, que, por todo lo que se ha demostrado, insiste en sembrar su propio descrédito con palabras vacías, mientras el mundo se retuerce entre lo tórrido y lo congelado, ajeno a las conspiraciones inventadas. Es necesario encontrar el punto de equilibrio. Los valores económicos, sociales y ambientales deben ir de la mano. Es necesario ser sostenible.

El ya fallecido Roger Scruton, que nos dejó el 12 de enero de 2020, hizo la advertencia:

"Hay personas que ven la política como una movilización social para lograr cierto objetivo; sin embargo, hay otros que lo ven como un procedimiento para resolver conflictos y conciliar intereses, sin un gran propósito incrustado. (...) Un gobierno sensible, el apoyo, no debe ir más allá, en sus objetivos, del arte de la reconciliación en la mediación de los objetivos de los ciudadanos" (Scruton, 2016).

El discurso político debe recurrir a la sostenibilidad, como dice Canotilho (2010, p. 8), visto como un valor autónomo y principio rector de los estados contemporáneos, presentándose como una herramienta importante y necesaria para las prácticas políticas necesarias capaces de prevenir el colapso.

\section{Conclusiones}

En vista de los innumerables datos recopilados sobre la evolución de la temperatura en el planeta, la incertidumbre no es sobre la realidad del cambio climático, sino cuál es el propósito de la campaña de desinformación difundida en gran medida en las redes sociales, quién está interesado y quién lo financia. No parece coherente que casi todos los expertos en el campo lleguen a la conclusión de que la amenaza es verdadera porque sirven a la agenda política de las ONG.

Por otro lado, es innegable que los políticos no tienen un compromiso intrínseco con la ciencia, sobre todo porque no están dedicados a ella y es probable que adopten la agenda de la influyente industria de los combustibles fósiles. "En general, lo que mueve a los políticos es el deseo de alcanzar o conservar el poder lo que, en pura lógica electoral, les lleva a intentar seducir cada cuatro años a sus potenciales electores con programas o promesas electorales en los que la necesidad de adoptar decisiones ambientales que impongan 'sacrificios' en forma de modificaciones de conductas, costes adicionales y/o alteraciones en los patrones económicos dominantes" (Real Ferrer, 2019, p. 17). Por lo tanto, los políticos tienden a no abordar los problemas ambientales para permanecer en el poder, lo cual es lamentable.

Aceptar la interdependencia ecológica y actuar para contener el proceso de degradación observado desde mediados del siglo XIX es más que un choque que fomenta la curiosidad académica entre la ciencia y la política, sino una cuestión de supervivencia. Ser económicamente viable, socialmente justo y ecológico es buscar la sostenibilidad (Boff, 2013, p. 43). El gran desafío para los que están en el poder es adoptar medidas, incluso si no son populares, a favor de la sostenibilidad, fomentando acciones que busquen el equilibrio social, económico y ambiental. 
La investigación científica no es infalible, pero siempre es necesaria para comprender los mayores problemas de la humanidad. Despreciarla y acosar a los investigadores no es un hecho histórico nuevo. Ha habido momentos en que esto ha sucedido en el mundo. La repetición de este procedimiento de abandono de la razón puede tener consecuencias más graves hoy, porque el agotamiento de la capacidad de la biosfera no era una realidad inminente en otras fases de la historia. Esta vez, relativizar con intenciones políticas las conclusiones alcanzadas por profesionales de ciertas áreas del conocimiento no es solo una estratagema éticamente cuestionable. Lo que está en juego no es solo una elección o las ganancias de grandes compañías.

Nuestro sistema democrático no parece estar preparado para tomar las decisiones necesarias para revertir el proceso de destrucción del medio ambiente. "Será necesario mejorarlo si no queremos seguir, como hasta ahora, sin dar eficaces respuestas a problemas de los que conocemos perfectamente sus soluciones, pero que no estamos dispuestos a adoptarlas" (Real Ferrer, 2019, p. 18). La irresponsabilidad política, la adulación de los patrocinadores electorales, el vaciamiento del debate, la reducción de los estímulos al conocimiento, pueden tener un precio impagable.

\section{Referencias}

Boff, Leonardo (2013). Sustentabilidade: o que é: o que não é. 2 ed. Petrópolis, RJ: Vozes.

Canotilho, José Joaquim Gomes (2010). O princípio da sustentabilidade como princípio estruturante do Direito Constitucional. Vol. VIII, n. 13, Barcelos, Portugal: Revista de Estudos Politécnicos.

Carvalho, Olavo de (2012). Palestra “O Enigma Quântico”. Recuperado de https://www.youtube.com/watch?v=bp2yoFJEaR0

Deutsche Welle (2020). Davos 2020: Trump Dismisses Environmental Concerns as 'Pessimism' at Climate-Focused WEF. Recuperado de https://www.ecowatch.com/davos-2020-trump-thunberg-2644880145.html

Dlouhy, Jennifer A. (2018). Climate changed: scientists to trump: 'zero reason' to expect a climate reversal. Recuperado de https://www.bloomberg.com/news/articles/2018-1015/trump-says-climate-change-no-hoax-but-will-change-back-again

Eler, Guilherme (2020). O que explica a temporada anormal de incêndios na Austrália. Recuperado de https://super.abril.com.br/ciencia/o-que-explica-a-temporada-anormalde-incendios-na-australia/

Embury-Dennis, Tom (2018). Trupm says he doesn't believe in climate change because 'air and water is at a record clean. Recuperado de https://www.independent.co.uk/news/world/americas/us-politics/trump-climatechange-air-water-clean-california-forest-fires-china-global-warming-a 8655396.html

Euronews (2019). Terra debate-se com temperaturas extremas. Recuperado de https://pt.euronews.com/2019/02/01/terra-debate-se-com-temperatura-extremas 
Friedman, Lisa (2018). I don't know that it's man-made', Trump says of climate change. It is. Recuperado de https://www.nytimes.com/2018/10/15/climate/trump-climate-changefact-check.html

García, Rocío (2019). Ministro brasileño: 'el cambio climático es un complot marxista'. Recuperado de https://larepublica.es/2019/01/13/ministro-brasileno-el-cambioclimatico-es-un-complot-marxista/

Hoegh-Guldberg, Ove, Jacob, Daniela y Taylor, Michael (coord.) (2018). Special report: global warming of $1.5^{\circ} \mathrm{C}$. Chapter-3. Recuperado de https://www.ipcc.ch/sr15/chapter/chapter-3/

Krugman, Paul (2018). Donald and the deadly deniers: climate policy is the ultimate example of Trumpian corruption. Recuperado de https://www.nytimes.com/2018/10/15/opinion/trump-climate-change-deniersrepublican.html

Martínez, Moisés B. (2018). El ministro de relaciones exteriores de Brasil dice que el cambio climático es un 'complot marxista'. Recuperado de https://www.pressdigital.es/textodiario/mostrar/1255377/ministro-relaciones-exteriores-brasil-dice-cambio-climaticocomplot-marxista

Programa de las Naciones Unidas para el Desarrollo, PNUD. (2008). Informe sobre desarrollo humano 2007-2008. La lucha contra el cambio climático: solidaridad frente a un mundo dividido. Madrid: Mundi-Prensa Libros.

Real Ferrer, Gabriel (2019). La complejidad de las decisiones ambientales. Revista Aranzadi de Derecho Ambiental, 44, pp. 11-18.

Roca, Ramón (2018). VOX 'pasa' del cambio climático: ni una referencia al medio ambiente en su programa electoral. Recuperado de https://elperiodicodelaenergia.com/voxpasa-del-cambio-climatico-ni-una-referencia-al-medio-ambiente-en-su-programaelectoral/

Sagan, Carl y Druyan, Ann (2006). O Mundo Assombrado pelos Demônios. São Paulo: Companhia das Letras.

Scruton, Roger (2016). Filosofia Verde: como pensar seriamente o planeta. São Paulo: É Realizações.

The Intergovernmental Panel on Climate Change (IPCC) (2018). Special report: global warming of $1.5^{\circ} \mathrm{C}$. Recuperado de https://www.ipcc.ch/about/

Universo On Line, UOL (2019). Olavo de Carvalho questiona se Terra orbita o Sol; o que diz a ciência? Recuperado de https://www.uol.com.br/tilt/ultimasnoticias/redacao/2019/01/09/o-que-a-ciencia-diz-sobre-a-terra-ser-o-centro-douniverso.htm 